\title{
How Magnetic Resonance Imaging is Helps in Diagnosis of Tuberculosis of Spine?
}

\author{
Kavita U Vaishnav ${ }^{1}$, Amisha G Patel ${ }^{2}$, Rahul Solanki ${ }^{3}$ \\ ${ }^{1}$ Associate Professor, Department of Radiology, L.G. Hospital, AMC MET Medical College, Maninagar, Ahmedabad- 380008, \\ ${ }^{2}$ Consultant, L.G.Hospital, AMC MET Medical College, Maninagar, Ahmedabad- $380008,{ }^{3}$ Consultant, L.G. Hospital, AMC MET \\ Medical College, Maninagar, Ahmedabad- 380008, India
}

Corresponding author: Dr. Amisha G Patel, L.G. Hospital, AMC MET Medical College, Maninagar, Ahmedabad- 380008, India

DOI: http://dx.doi.org/10.21276/ijcmsr.2019.4.3.31

How to cite this article: Kavita U Vaishnav, Amisha G Patel, Rahul Solanki. How magnetic resonance imaging is helps in diagnosis of tuberculosis of spine?. International Journal of Contemporary Medicine Surgery and Radiology. 2019;4(3):C141-C145.

\section{A B S T R A C T}

Introduction: Tuberculosis(TB) is a chronic granulomatous disease caused by mycobacterium tuberculosis, which is a major public health problem, especially in developing countries where poverty, malnutrition, overcrowding and the presence of drug resistance are the most common risk factors associated with tuberculosis. ${ }^{1,2}$ Magnetic resonance imaging (MRI) has the ability to provide excellent contrast resolution in all planes, making the diagnosis and its intra spinal involvement, marrow infiltration, intradural disease and to find skip lesions easily.

Study aimed to demonstrate, analyze and evaluate the magnetic resonance imaging as a preferred diagnostic tool for spinal tuberculosis and to promote early detection of spinal tuberculosis

Material and methods: A hospital based retrospective cross sectional study was done on 97 patients during the period from July 2017 to December 2018. MRI imaging of the spine was performed using a 1.5 Tesla Siemens MRI scan machine using a Phased Array surface coil.

Results: Lumbar vertebra (47\%) was the most commonly involved part of spine followed by dorsal, sacral and cervical segments. Endplate involvement (75\%) was most common type of presentation, followed by vertebral body and posterior elements involvement. Pre and para vertebral abscess is seen in $50 \%$ of the cases and psoas abscess in $19 \%$ patients. Epidural involvement was seen in $70 \%$ patients.

Conclusion: The higher contrast resolution of MR imaging is the best investigation showing spine involvement spread of infection into the adjacent structures, epidural collections and early diagnosis of subclinical infections.

Keywords: Spinal Tuberculosis, Para Spinal Collections, Epidural Collections

\section{INTRODUCTION}

Spine is the most common extra pulmonary site for skeletal tuberculosis. ${ }^{1-4}$ In developed countries, most cases of spinal tuberculosis are seen primarily in immigrants from tropical countries. Early detection of the disease by MRI and prompt treatment reduce the morbidity. It also prevents further neurological sequelae and spine deformities.

Various imaging modalities like, plain radiographs, bone scans, CT scans, myelograms and MR images are used for patients with spinal tuberculosis. The primary infection site is either a pulmonary lesion or an infection of the gastrointestinal or genitourinary system. The infection spreads to the vertebra through the perivertebral arteries, via batson's venous plexi. ${ }^{4}$ Usually two contiguous vertebras are involved, however, multiple vertebral involvements, skip lesions and single vertebra involvement is also seen. ${ }^{5}$

Spinal tuberculosis most commonly involves the lower thoracic spine and the lumbar spine, involvement of the cervical spine and sacrum is less common. ${ }^{5}$ Focal areas of osseous destruction involving the antero - inferior corners of the vertebral body are the initial plain film findings for tuberculous spondylitis. Involvement of the adjacent vertebral body results from penetration through the anterior longitudinal or posterior longitudinal ligament due to lack of proteolytic enzymes. Infection spread to involve two contiguous vertebral bodies, progressive vertebral body collapse result in the characteristic gibbous deformity and kyphosis of the spine commonly associated with tuberculosis of spine. ${ }^{5,6}$

The MR imaging features of spinal tuberculosis are believed to be diagnostic in the appropriate clinical setting. The multi planar imaging capability of MR imaging greatly improves the detection of vertebral destruction, pre and para vertebral abscesses, skip lesions, sub ligamentous spread of infection and epidural extension commonly associated with tuberculous spondylitis. ${ }^{7-9}$

As the involvement of vertebral body, inter vertebral disc space, extra and intra dural spinal cord occur, it was seen as hypo to intermediate signal intensity in T1W images, hyper intensity on T2W images and homogenous enhancement on gadolinium used post contrast study. 


\section{MATERIAL AND METHODS}

It was a Hospital based retrospective cross sectional study which enrolled subjects after obtaining an informed consent of the patients. 97 patients were taken for this study. Patients with back pain and neurological symptoms referred to our department for MRI spine study. Patients with recent trauma and known contraindication for MRI were excluded in our study.

Study time: Patients referred to the department of Radio diagnosis, L.G. Hospital, Maninagar, Ahmedabad for a period from July 2017 to December 2018.

MRI study was performed in the sagittal, coronal and axial planes. T1, T2, STIR, fat suppression and post contrast T1 sequences were taken. MRI imaging of the spine was performed using a 1.5 Tesla SIEMENS MRI scan machine using a Phased Array surface coil (figure-1-2).

\section{STATISTICAL ANALYSIS}

Data was analyzed using descriptive statistical analysis like proportions, percentage, etc.

\section{RESULTS}

In our study, out of 97 patients, the most common age group which presents with spinal tuberculosis was 20-29 years $-2^{\text {nd }}$ decade (35\%) followed by $3^{\text {rd }}$ decade. Among all the patients, male were more involved in 65 cases $(67 \%)$. Lumbar vertebra (47\%) was the most commonly involved part of spine followed by dorsal, sacral and cervical spine involvement in least cases.

Epidural collection is seen in (n-67) $70 \%$ cases. Intra dural involvement is seen in (n-6) $7 \%$ of patients.

Out of the total 97 patients, 76 patients (78\%) were having back pain. 35 patients (36\%) presented with neurological symptoms like paraplegia, weakness. 5 patients presented with spinal deformity. Other constitutional symptoms like, chronic cough, low grade fever, loss of appetite and weight loss were also present. Laboratory investigations like, ESR (Erythrocyte sedimentation rate), mantoux skin test, C reactive protein and $\mathrm{WBC}$ count were also done to reveal tubercular etiology. Out of total 97 patients, 62 were having present or past history of pulmonary tuberculosis.

Pre-para vertebral collections were seen in 49 patients (50\%)
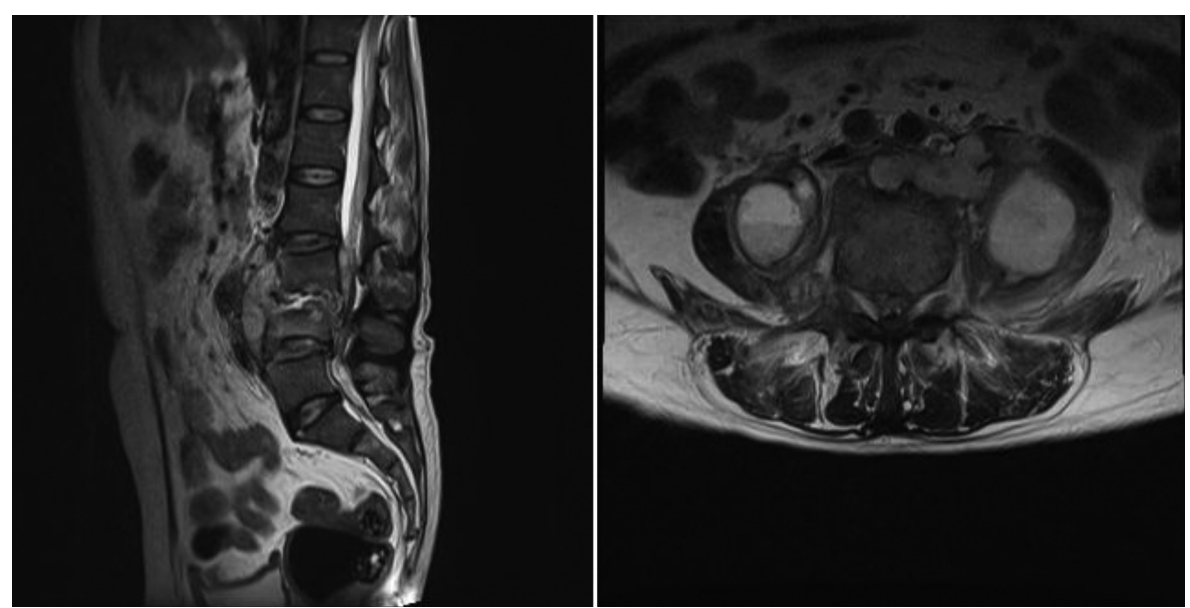

Figure-1a: Sagittal T2W image show hyper intense marrow signal intensities of L4-L5 vertebral body. Multi locular hypo intense collection is seen in pre-para vertebral location at L4-L5 level. Erosion of L4- L5 vertebral body is seen and upper end plate of L5 vertebral body also noted; Figure-1b: Axial T2W image show multi locular heterogeneously hyper intense signal intensities in pre-para vertebral collection at L4-L5 vertebral bodies which extends into neural foramina and anterior epidural space causing compression of thecal sac and bilateral nerve roots. Adjacent fibres of psoas muscles show hyper intense signal bilaterally, suggestive of edema.

\begin{tabular}{|l|c|c|}
\hline MRI findings in spinal tuberculosis & Number of patients & Percentage \\
\hline Endplate irregularity & 33 & $35 \%$ \\
\hline Disc space involvement & 30 & $31 \%$ \\
\hline Wedging and collapsed vertebrae & 29 & $29 \%$ \\
\hline Gibbus deformity & 14 & $15 \%$ \\
\hline Spinal nerve root and cord compression & 55 & $57 \%$ \\
\hline \multicolumn{2}{|c|}{ Table-1: MRI findings in spinal tuberculosis } \\
\hline
\end{tabular}

\begin{tabular}{|l|c|c|}
\hline Number of infected vertebrae & Number of patients & Percentage \\
\hline 1 vertebra & 2 & $65 \%$ \\
\hline 2 vertebrae & 62 & $57 \%$ \\
\hline $3-4$ vertebrae & 56 & $3 \%$ \\
\hline$>5$ vertebrae & 2 & \\
\hline \multicolumn{2}{|c|}{ Table-2: Number of vertebras involved } \\
\hline
\end{tabular}



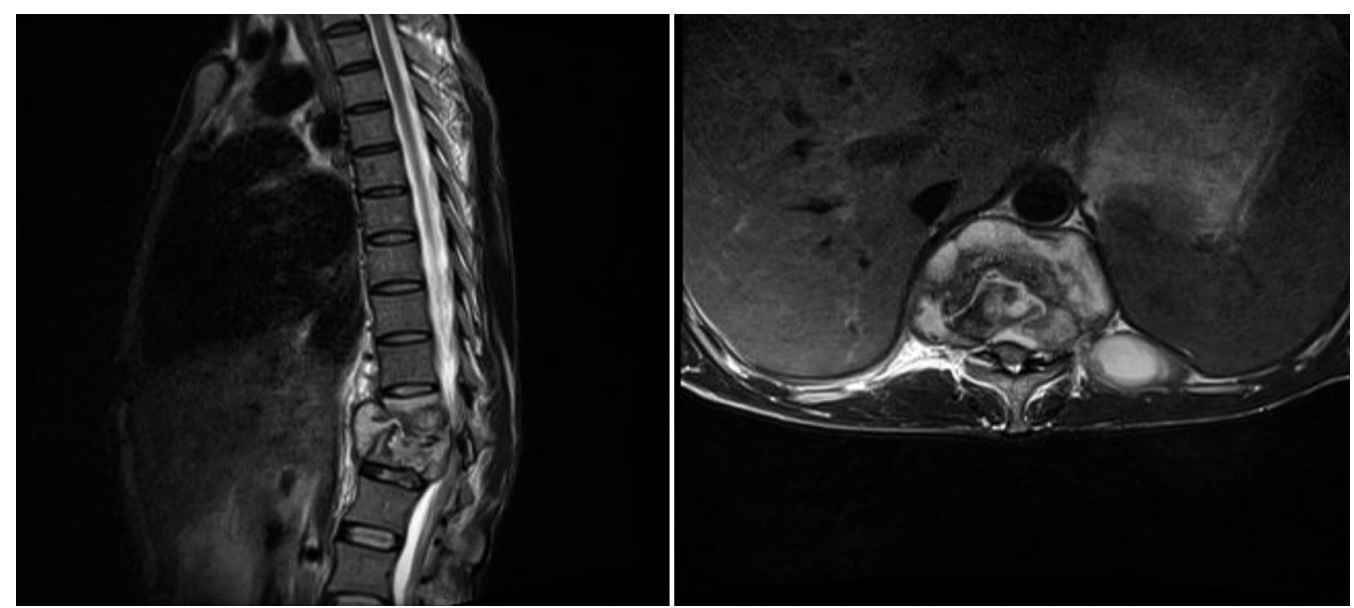

Figure-2a: Sagittal image shows hyper intense marrow signals involving D11-D12 vertebrae. Erosion involving D11- D12 vertebral body noted. Intervening disc space appears reduced. Epidural collection causing indentation and compression of spinal cord. Figure-2b: Axial image shows heterogeneously hyper intense loculated collection involving prevertebral and paravertebral space. Collection also seen in anterior epidural location compressing thecal sac and bilateral nerve roots.

and psoas abscesses were seen in 19 patients (19\%). Surgical interventions like USG guided percutaneous drainage and surgical debridement was done. Out of total 19 patients with psoas abscess USG guided percutaneous drainage was done in 16 patients. Of total 49 patients of pre-para vertebral collection, surgical debridement is done in 38 patients. Others were given only conservative management and antitubercular drugs.

Collection of sample from USG guided aspiration of pus or fluid taken under aseptic precautions and sent for acid fast bacilli (AFB) staining, AFB culture and sensitivity, aerobic culture and sensitivity. Out of 16 patients of psoas abscess 14 patients (16\%) were showed positive tubercular bacilli. Appropriate antibiotic is given and anti-tubercular drugs also continue afterwards.

Follow up MR study was done in 4 patients, in them improvement was noticed in form of reduction in size of prepara vertebral collection and marrow infiltration.

\section{DISCUSSION}

Tuberculosis is common infection occurs in India. Primary site for tuberculosis is the lung, lymph nodes, gastrointestinal or genitourinary system. Extra-pulmonary involvement is occurring via the hematogenous route through peri vertebral arteries, venous plexi and via the contiguous spread. $^{5,6}$ Tuberculosis infection is characterized by a delayed hypersensitivity immune reaction. Typically, the infection begins in the cancellous area of the vertebral body in para discal location or it involves the central or anterior part. The vertebral body becomes soft and weakened, so wedging of the body leads to kyphus and gibbus deformity. ${ }^{7,8}$ The spread of infection also occur beneath the anterior longitudinal ligament to involve adjacent vertebral bodies. The hypersensitivity reaction to mycobacterium results in pus formation which consists of langerhans' giant cells, epithelioid cells, and lymphocytes, caseous material and body fragments. The granulation tissue proliferates, producing thrombosis of vessels. ${ }^{6}$ The inflammatory cells cause destruction and necrosis of tissue and leads formation of para spinal abscess. The pus may be localized, or it may track along tissue planes.
The abscesses further extend to involve the spinal cord and produce epidural abscess which causes cord compression. ${ }^{7}$

MRI is the best imaging technique in TB spondylitis due to its multi planar capability, to demonstrate bone and adjacent soft tissue involvement, early bone marrow involvement or edema. It is used for assessment of spinal cord and neural involvement. MR is also better than CT to demonstrate epidural, meningeal and spinal cord involvement. CT scan is better in evaluation of bone destruction. ${ }^{8-10} \mathrm{~T} 1$-weighted images of the spine typically show decreased signal within the affected vertebral bodies, loss of disk height, and para spinal soft-tissue involvement.T2-weighted images often show increased signal within the areas of osseous involvement and adjacent soft tissue structures. Bony fragments and calcified foci within the abscess are called 'rice bodies' which are pathognomic for tubercular abscess. Contrast-enhanced MR sequences also shows the presence of a thick rim of peripheral enhancement around para spinal and intra osseous abscesses which is to be diagnostic of tuberculous spondylitis.

The spread of infection starting anteriorly and moving to involve opposing vertebrae via sub ligamentous spread is easily seen on MRI as heterogeneous enhancement of vertebral bodies and inter vertebral disc. Meningeal involvement which indicate active inflammation and rim enhancement around intra osseous and para spinal soft tissue abscesses, which are rarely seen in non-tubercular abscesses are best demonstrated on contrast enhanced MRI. ${ }^{11-12}$

Four different sites are found to involve in vertebral TB: para discal, anterior, central and posterior lesions. The majority of authors suggest that the onset of the spinal infection is para discal.

Para discal infection begins with in the metaphysis of vertebral body, later it causes erosion of the cartilaginous end plate, leading to disc space alteration due to the infection or may be due to disc herniation into the end plate. Due to osseous resorption, end plate demineralization with loss of cortical bone is observed. ${ }^{13}$

On T1-weighted sequences, vertebral bodies appear hypo intense while it appears hyper intense on both T2-weighted imaging and STIR sequence. Gadolinium used contrast 
study shows heterogeneous enhancement. This is the result of the replacement of bone marrow by inflammatory exudates, cells and hyperemia. Resurgence of the disease is most commonly seen in developed countries due to pandemic of Human immunodeficiency virus (HIV).

In the anterior pattern the infection starts just adjacent to the vertebral end plate of the vertebral body, it spreads to the adjacent vertebrae underneath the anterior longitudinal ligament. Sub ligamentous dissemination stripes the periostium and the anterior longitudinal ligament from the vertebral surface. Periostium stripping makes the avascular vertebrae more vulnerable to infection. There is scalloping of the antero lateral surface of the vertebral bodies ("gouge defect") occurs due to pressure effect form inflammatory exudates. It may mimic bone tumor, metastatic lymph nodes or even a thrombosed aortic aneurysm. Lastly, the progression of disease causes anterior vertebral wedging and collapse, leading to kyphosis ('hump'), typically seen in Pott's disease. MRI contrast imaging also show sub ligamentous and para spinal abscesses as ring like peripheral enhancement, preservation of the discs and abnormal signal involving multiple vertebral segments with heterogeneous signal intensity. ${ }^{14}$

In central type of lesions, the infection affects single vertebral body. The disc remains healthy, since nutrition is provided from the adjacent vertebra. In some cases infection progresses, the vertebral body collapses, and it can be difficult to differentiate it with malignancy. Infection spreads to the multiple vertebras or to the adjacent para spinal space. MRI shows hypo intense T1-weighted signal in a single vertebra and vertebral collapse with disc preservation. ${ }^{15}$

TB rarely affects the vertebral arch. This occurs in only $5 \%$ of cases. CT and MRI are useful to diagnose neural arch involvement. It most commonly involve cervical and upper dorsal spine. There is tendency to involve pedicle and lamina by tubercular infection. However, pyogenic infection involves facet joints. ${ }^{16-20}$

Paraplegia in patients with active infection may be caused by mechanical pressure on the cord by tubercular abscesses, granulomatous tissue, debris, gibbus or subluxation of vertebrae. Compression of the spinal cord results in loss of neurons, gliosis and demyelination of cord in the damaged segment. Intrinsic changes in the spinal cord such as inflammatory edema or direct involvement of meninges and spinal cord by tuberculous infection leads to paraplegia. The changes in the spinal cord interpreted as edema of the cord, myelomalacia, atrophy of the cord. Edema seen as hyper intense signal on T2 weighted images with thecal compression. Myelomalacia shows hypo intense signal on T1W images. Clinical history is required to differentiate cord edema and myelomalacia.

At present, management of spine $\mathrm{TB}$ is done by systemic treatment with anti-tubercular medications before and after the surgical debridement, the careful debridement of entire focus of infection and the appropriate method to reconstruct for spinal stability are the key aspect in the treatment of tuberculosis. In patients without neurological deficit, medical therapy is the treatment of choice. While in cases with neurological involvement combination of both medical and surgical treatment provide best results. Laminectomy is useful in patients with posterior complex disease.

Combinations of rifampicin, isoniazid, ethambutol and pyrazinamide, streptomycin and other newer drugs for a period of six to eighteen months along with surgical intervention according to treating consultant are the recommended treatment for spinal TB. Early diagnosis of TB is very important as adequate medical treatment prevent further severe complications.

Differentiation between tubercular spondylitis from pyogenic spondylitis is important because proper treatment of the different spinal infection can reduce the rate of neurological disability and functional impairment. MRI has been shown to be accurate in differentiating tuberculosis spondylitis from pyogenic spondylitis. The presence of a well-defined para spinal abnormal signal, a thick rim enhancement of abscess wall with internal calcifications, sub ligamentous spread to three or more vertebral levels, skip lesions and disc preservations are more suggestive of tuberculosis spondylitis than pyogenic spondylitis. ${ }^{21}$

The differential diagnosis of the tuberculous spine is pyogenic and fungal infections, brucellosis, sarcoidosis, metastasis, and lymphoma. Typically, infectious spondylitis is characterized by involvement of inter vertebral disk. Proper history of disease chronicity and its slow progression is useful to diagnose tubercular spondylitis.

In brucellosis infections, gas within the disc, minimal para spinal abscess and absence of gibbus deformity is seen. It most commonly involves lumbar spine.

Serial MRI scan helps in suggesting response to treatment. Appearance of sclerosis and the fatty changes in bony tissue, ankylosis and reduction in epidural and para spinal collection, suggests resolution of disease. Increase in destruction of vertebral body with increase in associated collection would suggest drug resistant and thus indicates changes in management. Soft tissue involvement in form of pre- para vertebral and psoas abscess is well assessed by MRI in guiding the surgical intervention and during follow up.

\section{Limitation of the study}

Calcification which is pathognomic for tubercular infection and any bony fragments not properly evaluated by MRI.

All suspected patients for Pott's spine and those known patients who require follow up scans could not afford for MRI scan. Hence, the cost of the modality and the patient affordability for MRI continues to be a drawback.

\section{CONCLUSION}

MRI is the modality of choice for early detection of spinal tuberculosis, characterizing the lesion, detection and staging of the disease and follow up to evaluate the success of treatment and detecting complications, like spinal cord involvement and the extent of the epidural collections. It provides necessary information to the surgeon about diagnostic as well as therapeutic management of spinal tuberculosis.

\section{REFERENCE}

1. DD, Bradley WG. Magnetic Resonance Imaging. $3^{\text {rd }}$ edition. Mosby;s Chapter 86, Thoracic spine, 1999; p. 
1851-82.

2. Burrill J, Williams CJ, Bain G, Conder G, Hine AL, Misra RR. Tuberculosis: A Radiologic Review. Radiographics, 2007; 27(5): 1255-73.

3. Yao DC, Sartoris ADJ. Musculoskeletal tuberculosis. Radiol Clin N Am. 1995;35(4):679-89.

4. Moore SL, Rafi M. Imaging of musculoskeletal and spinal tuberculosis, Radiol Clin N Am. 2001;39(2):32942.

5. Bhan S, Nag HL. Skeletal tuberculosis. In: Sharma SK, Mohan A(Eds). Tuberculosis. Jaypee Publishers; 200. pp.237-60.

6. Resnik D. Osteomyelitis, septic arthritis and soft tissue infection: mechanism and situations. In: ResnickD (Ed). Diagnosis of bone and joint Disorder, $4^{\text {th }}$ edition, Philadelphia: WB Saunders; 2002.pp. 2377-480.

7. Stabler A, Resier MF. Imaging of spinal infection. Radiol Clin N Am. 2001; 39(1): 115-35.

8. Maiuri F, Iaconetta G, Gallicchio $B$, et al. Spondylodiscitis Clinical and Magnetic Resonance Diagnosis. Spine. 1997;2(2):1741-6.

9. Sharif HAS. Role of MR imaging in the management of spinal infections. AJR Am J Roentgenol. 1992;158:1333

10. Kaila R, Malhi AM, Mahmood B, Saiffuddin A. The incidence of multiple level noncontiguous vertebral tuberculosis detected using whole spine MRI. J Spinal Disord Tech. 2007;20 (1):78-81.

11. SharifHAS, Clark DC,Aabed MY, et al. Granulomatous spinal infections MR imaging radiology. 1990;177 (4):101-7.

12. Palle L, Reddy MB, Reddy KJ. Role of magnetic resonance diffusion coeeficient values in the evaluation of spinal tuberculosis in Indian patients. Indian J Radiol Imaging. 2010;20(4):279-83.

13. Weaver P, Lifeso RM. The radiological diagnosis of tuberculosis in the adult spine. Skeletal Radiol. 1984;12 (6):178-86.

14. Tuli SM. Tuberculosis of the craniovertebral region. Clin Orthop Res 1974; 104:209.

15. Golding FC. Tuberculosis of bone. In: Shanks CS Kerly P (Eds). A textbook of x-ray Diagnosis by British Authors. $3^{\text {rd }}$ edition. 1959. Pp. 274-89.

16. Rahman NU, Jamjoom A, Jamjoom ZAB, et al. Neural arch tuberculosis: Radiological features and their correlation with surgical findings. $\mathrm{Br} \mathrm{J}$ Neurosurg. 1997;11(1):32-8.

17. Loke TKL, Ma HT, Chan CS. Magnetic resonance imaging of the tuberculous spinal infection. Australas Radiol. 1997;41 (2):7-12.

18. Kumar K. Clinical study and classification of posterior spinal tuberculosis. Int Orthop. 1985:9 (1):147-52.

19. Raglan R, Abdelwahan F, Braffman B, et al. Posterior spinal tuberculosis-a case report. AJNR. 1990;11 (5):612.

20. Jain R, Sawhney S, Berry M. Computed tomography of vertebral tuberculosis: patterns of bone destruction. Clinical Radilology. 1993;47 (6):196-9.

21. Sharif HAS, Clark DC, et al. Brucellar and tuberculous spondylitis: comparative imaging features. Radiology. 1989;155 (3):419-25.

22. Bureau NJ, Cardinal E. Imaging of musculoskeletal and spinal infections in AIDS. Radiol Clin North Am 2001;39 (1):343-355

23. Moore SL, Rafii M. Imaging of musculoskeletal and spinal tuberculosis. Radiol Clin North Am 2001;39 (5):329-342

24. Hodgson AR, Skinsnes OK, Leong CY. The pathogenesis of Potts paraplegia. J Bone Joint Surg Am 1967;49 (4):1147-1156

25. Ho EKW, Leong JCY. The pediatric spine: principles and practice. In: Weinstein SL, ed. Tuberculosis of the spine, 3rd Ed. New York: Raven, 1994: 837-849

26. Cotran SR, Kumar V, Tucker C. Robbins pathologic basis of disease, 6th ed. Philadelphia: Saunders, 1999:349-352

27. Smith AS, Weinstein MA, Mizushima A, et al. MR imaging characteristics of tuberculous spondylitis vs vertebral osteomyelitis. AJNR 1989;10 (1):619-625

28. Ahmadi J, Bajaj A, Destian S, Segall HD, Zee CS. Spinal tuberculosis: atypical observations at MR imaging. Radiology 1993;189 (2):489-493

29. Sharma A, Goyal M, Mishra NK, Gupta V, Gaikwad SB. MR imaging of tubercular spinal arachnoiditis. AJR 1997;168 (2):807-812.

30. Dunn R, Zondagh I, Candy S. spinal tuberculosis: magnetic resonance imaging and neurological imparement. Spine (Phila Pa 1976) 2011;36(6):469-73.

\section{Source of Support: Nil; Conflict of Interest: None}

Submitted: 12-06-2019; Accepted: 20-08-2019; Published online: 04-09-2019 